\title{
Numerical analysis of the strain values obtained by FBG embedded in a composite material using assumptions about uniaxial stress state of the optical fiber and capillary on the Bragg grating.
}

\author{
Valerii P. Matveenko \\ Institute of Continuous Media Mechanics UB RAS \\ mvp@icmm.ru, bttp://orcid.org/0000-0003-2787-6558
}

Natalia A. Kosheleva

Perm National Research Polytechnic University

nataly.kosheleva@gmail.com, bttp:/ /orcid.org/0000-0002-8760-2957

Grigorii S. Serovaev, Andrey Yu. Fedorov

Institute of Continuous Media Mechanics UB RAS

serovaev@icmm.ru, bttp:/ / orcid.org/0000-0003-0312-8088

fedorov@icmm.ru, http://orcid.org/0000-0001-8239-3386

\begin{abstract}
One of the issues in the strain measurement with the help of fiber-optic sensors based on Bragg gratings is the calculation of the strain based on the information from the sensor and its correspondence to the material strain. Relationships between the data measured by the sensor and the strain in the Bragg grating have a unique solution under the condition of uniaxial stress state of the fiber, which are not fulfilled when the fiber is embedded in the material. The paper presents the results of numerical simulations with an example of a model problem for a polymer composite material with an embedded optical fiber, which can be surrounded by a resin pocket. The presented results allow to estimate the error of the strain values calculated on the basis of uniaxial stress state assumption of the optical fiber. Based on numerical calculations, parameters of a capillary on an optical fiber in the Bragg grating zone are estimated, ensuring uniaxial stress state of the fiber in the Bragg grating zone. To ensure these conditions, the cross section of the capillary in an isotropic material is a circle, and in an anisotropic material, good results can be obtained with an elliptical cross section.
\end{abstract}

KEYwORDS. Single-mode optical fiber; FBG; polymer composite material; sensor embedded in the material; capillary.

\section{OPEN ACCESS}

Citation: Matveenko, V., Kosheleva, N., Serovaev, G., Fedorov, A., Numerical analysis of the strain values obtained by FBG measurements embedded in a composite material using assumptions about uniaxial stress state of the optical fiber and capillary on the Bragg grating, Frattura ed Integrità Strutturale, 49 (2019) 177-189.

Received: 09.04.2019

Accepted: 16.05.2019

Published: 01.07.2019

Copyright: (C) 2019 This is an open access article under the terms of the CC-BY 4.0, which permits unrestricted use, distribution, and reproduction in any medium, provided the original author and source are credited. 


\section{INTRODUCTION}

$\mathrm{N}$

owadays fiber-optic sensors successfully compete and in some cases displace piezoelectric, acoustic, thermal, electromagnetic, electrical devices for measuring physical quantities. This is a consequence of the unique properties of fiber-optic sensors: insensitivity to electromagnetic effects and to radiation; no need for power supply near the sensor; ability to work in a wide temperature range. The fiber is both a sensor and information transmission channels, which makes it possible to use fiber-optic systems for objects of large size successfully. Fiber optic sensors are based on the use of various physical effects. In this paper, the widely used fiber optic sensors based on Bragg gratings written in a standard single-mode optical fiber for strain measurements are considered. Examples of their successful applications in various structures and industries are contained in the Refs. [1-6].

For structural health monitoring one of the most demanded are products made of polymer composite materials (PCM). The known methods do not provide a guaranteed solution to the corresponding issues while the range and areas of application of PCM are constantly expanding. The small dimensions of the optical fiber and the manufacturing process of PCM products make it possible to embed optical fibers into PCM structure. The use of fiber-optic strain sensors (FOSS) embedded into material discovers fundamentally new possibilities for structural health monitoring of constructions during their operation, including the stage of manufacturing process of material. Optical fibers can be embedded between different layers of the composite material, thereby forming a network of optical fibers inside material.

A number of problems arise when FOSS based on Bragg gratings embedded into PCM are used. One of the most difficult tasks is related to the evaluation and search for options to ensure the reliability of strain values calculated on the basis of a physical quantity recorded by a sensor. Great opportunities to solve this problem are associated with mathematical modeling. A significant number of works are devoted to this area. The main essence of the problem is that the wellknown relations between the physical quantity recorded by the sensor and the components of the strain tensor in the zone of Bragg gratings [7] have a unique solution only under the condition of uniaxial stress state in an optical fiber. Optical fiber embedded in a material, as a rule, operates under complex stress state. It should also be noted that in order to make reasonable use of FOSS, it is necessary to ensure the coincidence of strains along the fiber in the material and in the Bragg grating zone of the optical fiber. In the given in Ref. [8], it is noted that the embedded fiber Bragg grating (FBG) operates under complex stress state and, therefore, for the FBG it is necessary to introduce calibration coefficients for the corresponding strains. In this case, studies have shown that the calibration of the transverse strains is difficult to control. Calibration problems are discussed in detail in Refs. [9,10]. In Ref. [11], it is noted that large radial strains lead to a large deviation of the measured strain by embedded FBGs. It is noted that after embedding into the material, each sensor must be calibrated or, for the embedded sensor, the strain tensor must have a dominant component along the fiber axis. As a constructive solutions to obtain reliable information about the axial components of the strain tensor, it is proposed in Refs. [12,13] to use a pair of micro-structured FBGs that are precisely oriented relative to each other. A sensor model is proposed that is experimentally tested on layered composites. In Ref. [14], it is proposed to introduce a transfer matrix based on numerical modeling, the components of which establish the connection of strains in the material and fiber and are calculated based on consideration of three loading options: tension along the optical fiber, out of plane transverse load and transverse load in the plane of the specimen.

In Ref. [15], the problem of estimating the strain transfer from the host material to the embedded sensor is also considered. Experimental data show that under certain types of loading strong birefringent effects are present. In order to eliminate the effect of transverse strains on the embedded optical fiber measurements, the authors use a capillary in the region of the Bragg grating. Such scheme leads to uniaxial stress state of the grating and the relationship between the longitudinal and transverse components of the strain tensor through the Poisson coefficient. In order to ensure the sensitivity of the Bragg grating to compressive strain, a preliminary tension of the optical fiber is carried out. Encapsulation of the Bragg grating in the capillary ensures the single peak on the reflected spectrum after the implementation of the technological process of composite material production. The results of fairly accurate measurements of the strain field in carbon-fiber specimens are demonstrated. Another example of the use of a capillary in the region of the Bragg grating is given in Ref. [16], in which the use of a capillary is aimed at increasing the temperature sensitivity of a fiber-optic sensor. The authors demonstrate the applicability of the proposed approach to various FBGs enclosed in a zinc capillary and various binder materials. An example of the use of a transfer matrix is discussed in Ref. [17], where the results based on numerical and experimental data are given. The importance of converting measured strains into real strains, which can differ significantly from each other, is demonstrated.

This paper proposes a method for the numerical estimation of the error of strain values determined by formulas based on the assumption of uniaxial stress state of an optical fiber. The results are presented when the PCM is represented by a 
layered model and a homogeneous medium model. A variant of the embedded optical fiber in the presence and absence of a resin pocket is considered. The use of a capillary in the area of the Bragg grating is proposed as a constructive solution to ensure the use of the embedded FOSS on Bragg gratings for various types of loading. Based on numerical calculations, the capillary parameters are estimated, which ensure uniaxial stress state of the fiber in the Bragg grating zone and the correspondence of the strains in the material and the strains calculated on the basis of the physical quantity measured by the FBG.

\section{NUMERICAL ANALYSIS OF STRAINS, CALCULATED BASED ON EMBEDDED FBG MEASUREMENTS}

he measurement of strain by sensors based on Bragg gratings written in a standard single-mode optical fiber is implemented according to the diagram presented in Fig. 1.

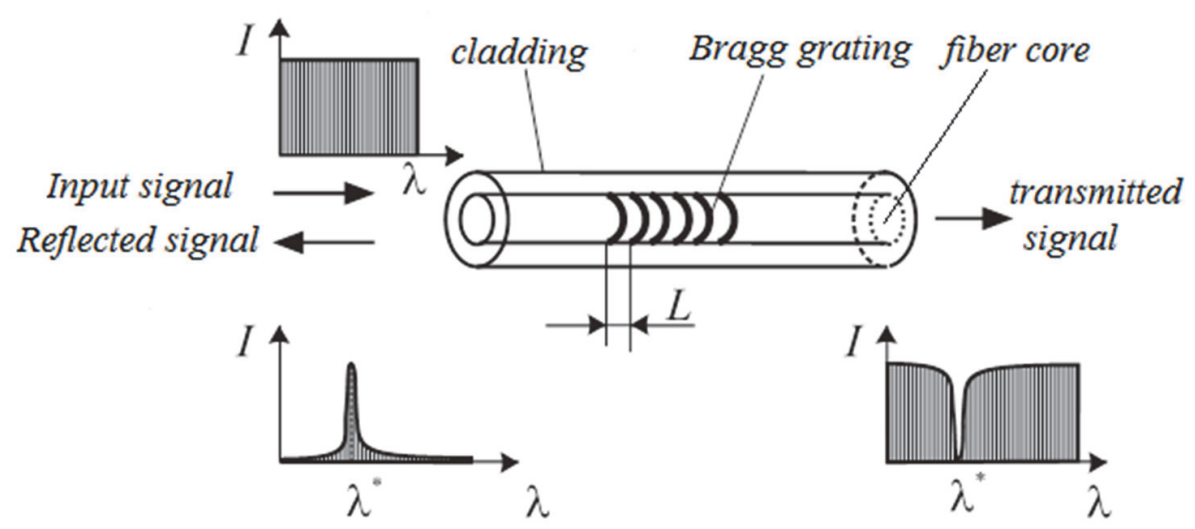

Figure 1: The principle of FBG operation.

The interrogator sends a broadband optical signal over an optical fiber in a given wavelength range. Part of this signal is reflected on the Bragg grating. The main part of the reflected signal determines the resonant wavelength of the reflected spectrum. This wavelength $\lambda^{*}$ is proportional to the effective refractive index $n$ of the optical fiber in the area of the grating and the geometric length of the period of the Bragg grating L [8]:

$$
\lambda^{*}=2 n L
$$

The scheme of strain measurement by a FBG sensor embedded into PCM is shown in Fig. 2.

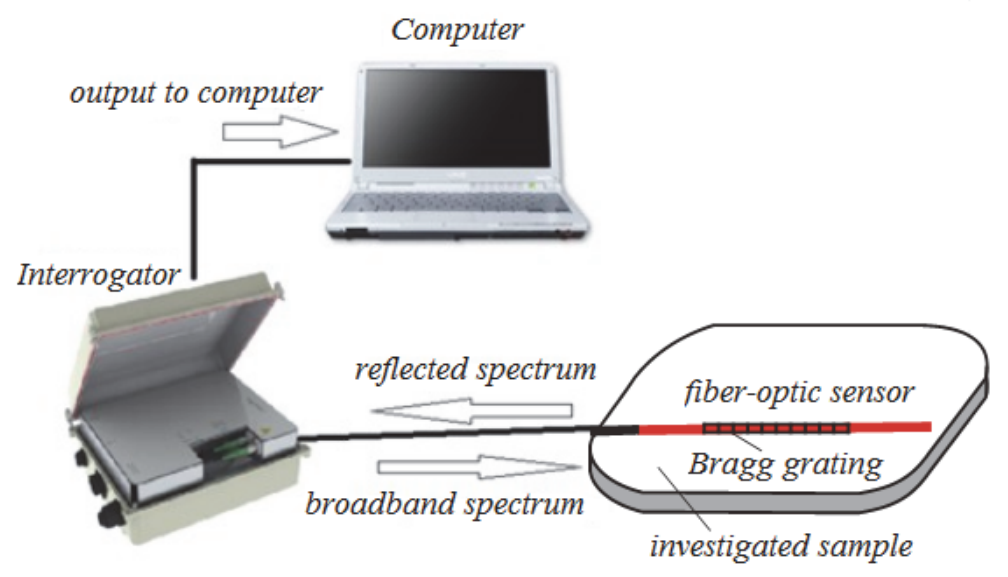

Figure 2: The scheme of strain measurement by a FBG sensor. 
When FOSS interacts with a deformable material, a change in the Bragg grating length occurs. This leads to a change in the wavelength of the reflected spectrum $\Delta \lambda$, which is recorded by the interrogator. The relationship between the changes in the Bragg wavelength and the strain of the fiber in the Bragg grating zone is determined by the relations [8]:

$$
\begin{aligned}
& \frac{\Delta \lambda_{1}}{\lambda^{*}}=\varepsilon_{3}-\frac{1}{2} n^{2}\left(p_{11} \varepsilon_{1}+p_{12}\left(\varepsilon_{2}+\varepsilon_{3}\right)\right) \\
& \frac{\Delta \lambda_{2}}{\lambda^{*}}=\varepsilon_{3}-\frac{1}{2} n^{2}\left(p_{11} \varepsilon_{2}+p_{12}\left(\varepsilon_{1}+\varepsilon_{3}\right)\right)
\end{aligned}
$$

where $\varepsilon_{3}$ - is the strain along the fiber, $\varepsilon_{1}, \varepsilon_{2}$ - are the principle strains in the plane perpendicular to the optical fiber, $\Delta \lambda_{1}=\lambda_{1}-\lambda^{*}, \Delta \lambda_{2}=\lambda_{2}-\lambda^{*}$ - are the differences in the values of the resonant wavelengths of the reflected spectrum at the current $\lambda_{1}, \lambda_{2}$ and initial $\lambda^{*}$ moments of time, $p_{11}, p_{12}$-are the Pockels coefficients.

Under uniaxial stress state of an optical fiber, which is free from interaction with the environment, the relation between strain components can be expressed as $\varepsilon_{1}=\varepsilon_{2}=-v \varepsilon_{3}$, where $v$ is the Poisson's ratio of the optical fiber. In this case $\Delta \lambda_{1}=\Delta \lambda_{2}=\Delta \lambda$ and

$$
\frac{\Delta \lambda}{\lambda^{*}}=\left(1-\frac{n^{2}}{2}\left(p_{12}-v\left(p_{11}+p_{12}\right)\right)\right) \varepsilon_{3}
$$

or

$$
\varepsilon_{3}=\frac{1}{k} \cdot \frac{\Delta \lambda}{\lambda^{*}}
$$

For quartz optical fiber with parameters $p_{11}=0.113, p_{12}=0.252, n=1.5, v=0.17$, the coefficient $k \sim 0.79$.

When the fiber-optic sensor is embedded in a polymer composite material or even connected in some way with the surface of the material, for example, by gluing, a complex-stressed state in the sensor as a result of the interaction with the material, will be realized. There will be strains $\varepsilon_{1}, \varepsilon_{2}, \varepsilon_{3}$ that need to be identified on the basis of experimental information, namely the values of $\frac{\Delta \lambda_{1}}{\lambda^{*}}$ and $\frac{\Delta \lambda_{2}}{\lambda^{*}}$. From Eq. (2) it follows that if $\varepsilon_{1} \neq \varepsilon_{2}$, we have two equations with three unknowns, and when $\varepsilon_{1}=\varepsilon_{2}$ one equation with two unknowns.

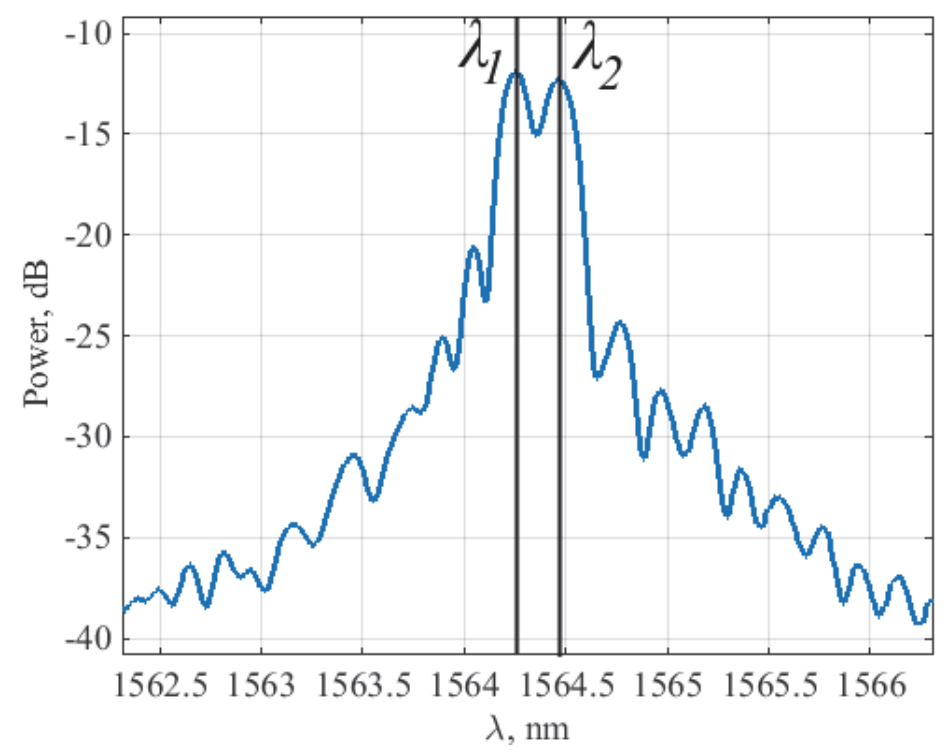

Figure 3: The reflected spectrum with two peaks of FOSS embedded in PCM. 
The presence in the reflected spectrum of two resonant wavelengths as a result of the interaction of the Bragg grating with the surrounding material is noted as an experimental result, for example, in Refs. [8,18]. The following result can be presented as an example. The FOSS with a Bragg wavelength of $1565 \mathrm{~nm}$ was embedded in the middle part of the specimen (a plate with a size of $50 \times 200 \times 5 \mathrm{~mm}$ ), manufactured by an autoclave method and consisting of 20 layers of fiberglass prepreg (VPS-48). After the sample is manufactured, the process-induced strains take place in it [19]. As a result, the embedded FBG is under the influence of these strains, and two peaks are observed in the reflected spectrum (Fig. 3). Thus, a direct relation between the experimental information $\Delta \lambda / \lambda^{*}$ and the strain component $\varepsilon_{3}$ along the fiber takes place only under the uniaxial stress state of the Bragg grating.

Numerical simulations allow to estimate the limits of applicability of Eq. (4). As a model object, parallelepiped with integrated optical fiber (Fig. 4) was considered with different loading options (normal displacements along the side faces $\left.u_{x}=u_{o} ; u_{y}=u_{o} ; u_{z}=u_{o}\right)$.

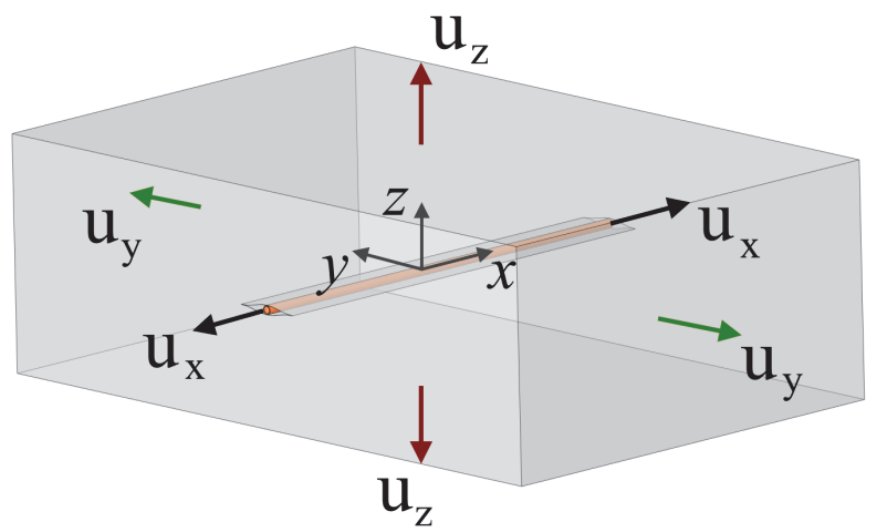

Figure 4: The calculation scheme for material with embedded FOSS.

The parameters of the polymer composite material related to the $x, y, z$ axes and optical fiber are shown in Tab. 1 and Tab. 2. The fiber has a diameter of $0.124 \mathrm{~mm}$ and a protective polyimide coating of $0.012 \mathrm{~mm}$ thick.

\begin{tabular}{cccccccccc}
\hline Material & $\begin{array}{c}E_{X}, \\
\mathrm{GPa}\end{array}$ & $\begin{array}{r}E_{Y}, \\
\mathrm{GPa}\end{array}$ & $\begin{array}{c}E_{Z}, \\
\mathrm{GPa}\end{array}$ & $v_{X Y}$ & $v_{Y Z}$ & $v_{Z X}$ & $\begin{array}{c}G_{X Y}, \\
\mathrm{GPa}\end{array}$ & $\begin{array}{c}G_{Y Z}, \\
\mathrm{GPa}\end{array}$ & $\begin{array}{c}G_{Z X}, \\
\mathrm{GPa}\end{array}$ \\
$\begin{array}{c}\text { unidirectional prepreg } \\
\begin{array}{c}\text { effective mechanical characteristics } \\
\text { of the layer stacking }\end{array}\end{array}$ & 158.5 & 8.96 & 8.96 & 0.32 & 0.45 & 0.32 & 4.6 & 3.0 & 4.6 \\
\begin{tabular}{c} 
(1). \\
\hline
\end{tabular} & 84.1 & 10.7 & 0.035 & 0.45 & 0.45 & 4.3 & 3.5 & 3.5 \\
\hline
\end{tabular}

Table 1: Mechanical properties of the unidirectional prepreg and effective mechanical characteristics of the layer stacking.

\begin{tabular}{ccc}
\hline Material & E, GPa & $v$ \\
quartz & 71.4 & 0.17 \\
polyimide & 2.5 & 0.35 \\
epoxy & 2.9 & 0.36 \\
\hline
\end{tabular}

Table 2: Mechanical characteristics of optical fiber and epoxy.

In numerical experiments, PCM was considered on the basis of a layered and homogeneous models (Fig. 5). 

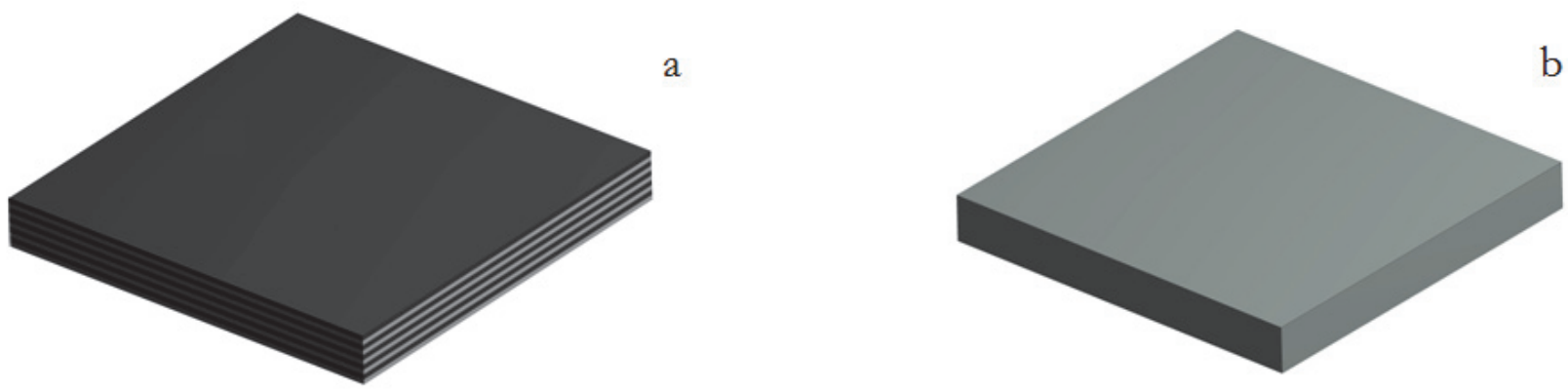

Figure 5: Variants of composite material models: layered (a) and homogeneous (b).

In a layered model, the PCM is represented as a layer stacking, each of which is a homogeneous anisotropic body. This model allows to take into account the different orientation of the layers. PCM in the framework of a homogeneous model is represented as a homogeneous anisotropic elastic body with effective mechanical characteristics. The optical fiber embedded between the PCM layers, retains its geometry. Wherein the prepreg layers in the vicinity of the fiber can be bent, which leads to the pocket formation filled with polymer matrix. This sub-area is a technological defect called a resin pocket. The geometry of a resin pocket significantly depends on the type of prepregs and their stacking options. For unidirectional prepregs, there may be various options associated with the formation of a resin pocket. In particular, if there are two layers [0/0] in the layer stacking and the fiber is laid between these layers then for this variant the resin pocket is not formed. The option of having two layers [90/90] in a laminated composite material and an optical fiber embedded between these layers leads to the formation of the maximum resin pocket, with all other factors equal. The geometry of the resin pocket (Fig. 6) obtained by experimental and numerical methods is shown in Ref. [20].

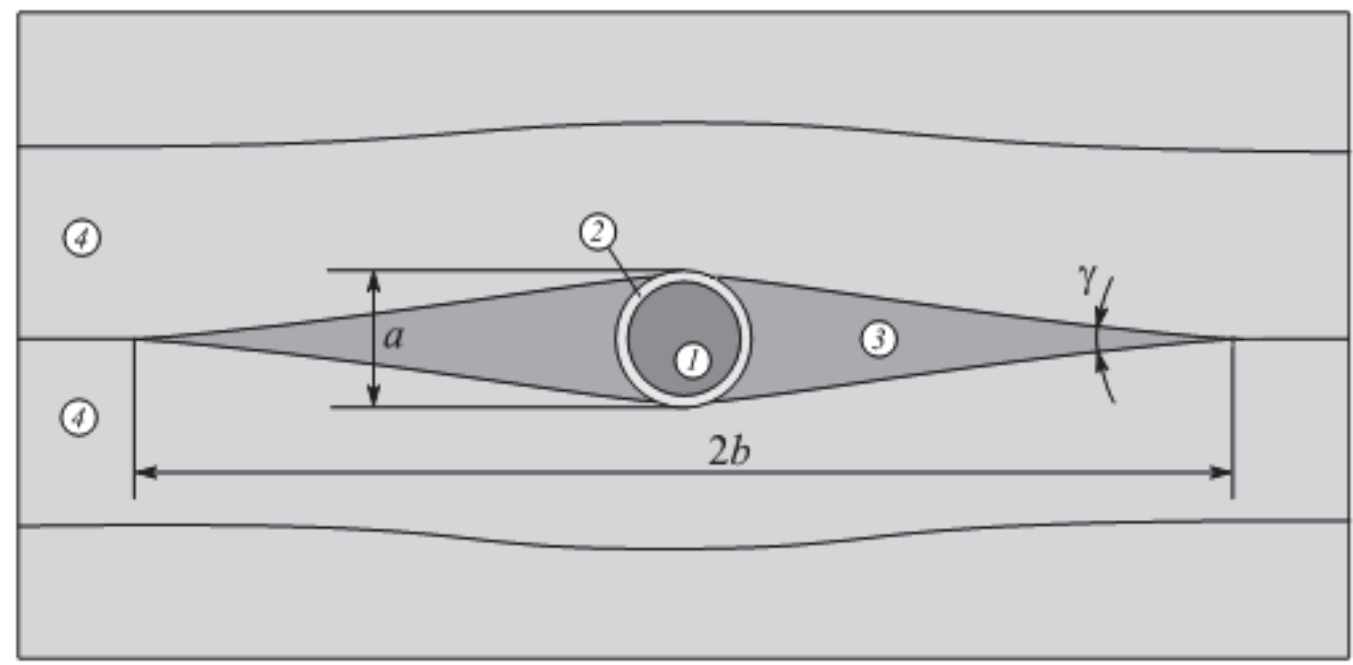

Figure 6: Polymer composite material with optical fiber and resin pocket: optical fiber (1); optical fiber coating (2); a resin pocket (3); polymer composite layer (4).

In this paper, the variants of a layered model without a resin pocket when the optical fiber is placed between the [0/0] layers and with resin pocket when the fiber is placed between the [90/90] layers are considered. Also, variants with and without a resin pocket are considered for a homogeneous model. Cross-sections of the corresponding model objects are shown in Fig. 7.

Following the conclusions given in Ref. [21], the following size of the resin pocket is taken $b=8 R_{1}$. The calculations were carried out by the finite element method in a three-dimensional formulation using mesh that is refined in the zone of stress concentration. Fig. 8 shows the finite element discretization of the cross section of the model. The accuracy of calculations was estimated on the basis of an analysis of the convergence of solutions with an increase in the number of elements. 
Layered model without the formation of a resin pocket [0/0] Homogeneous model without the formation of a resin pocket
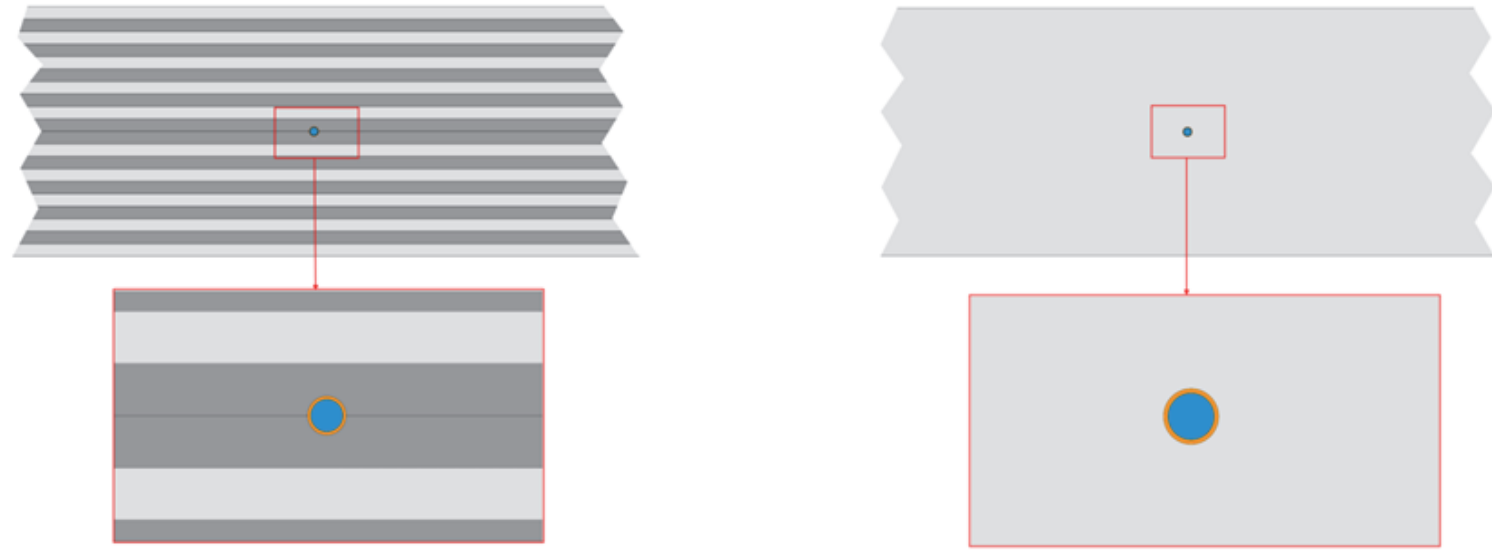

Layered model with the formation of a resin pocket $[90 / 90]$

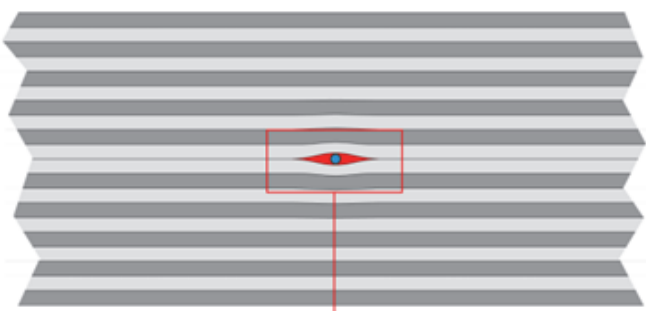

Homogeneous model with the formation of a resin pocket
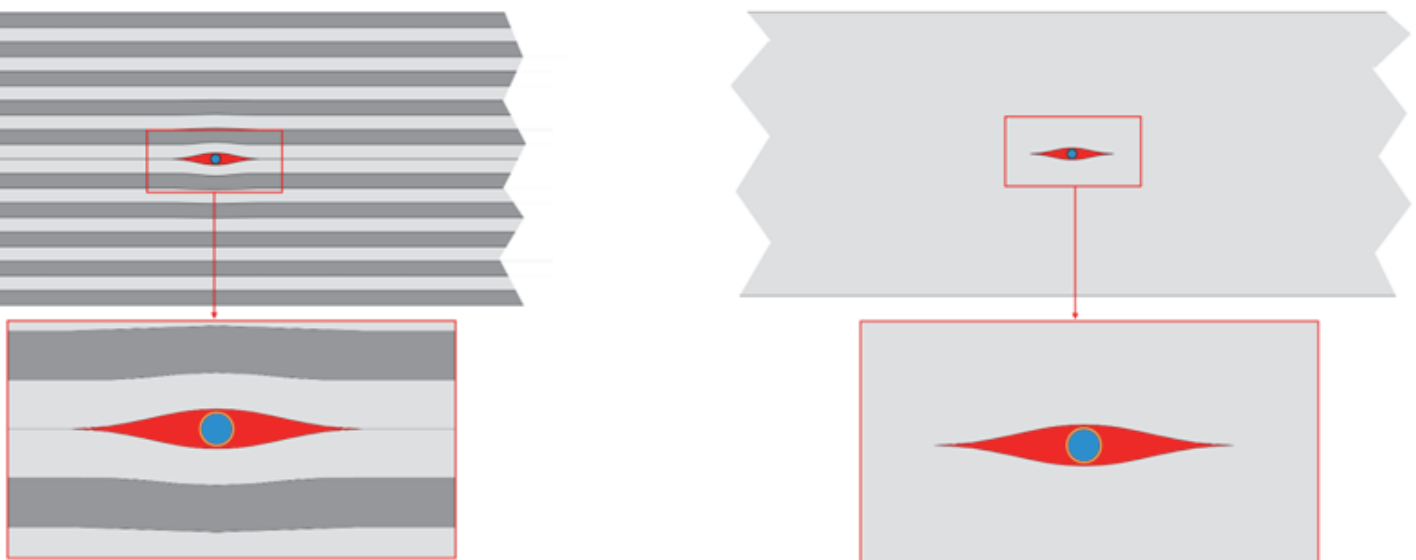

Figure 7: Variants of the representation of a polymer composite material with embedded optical fiber.

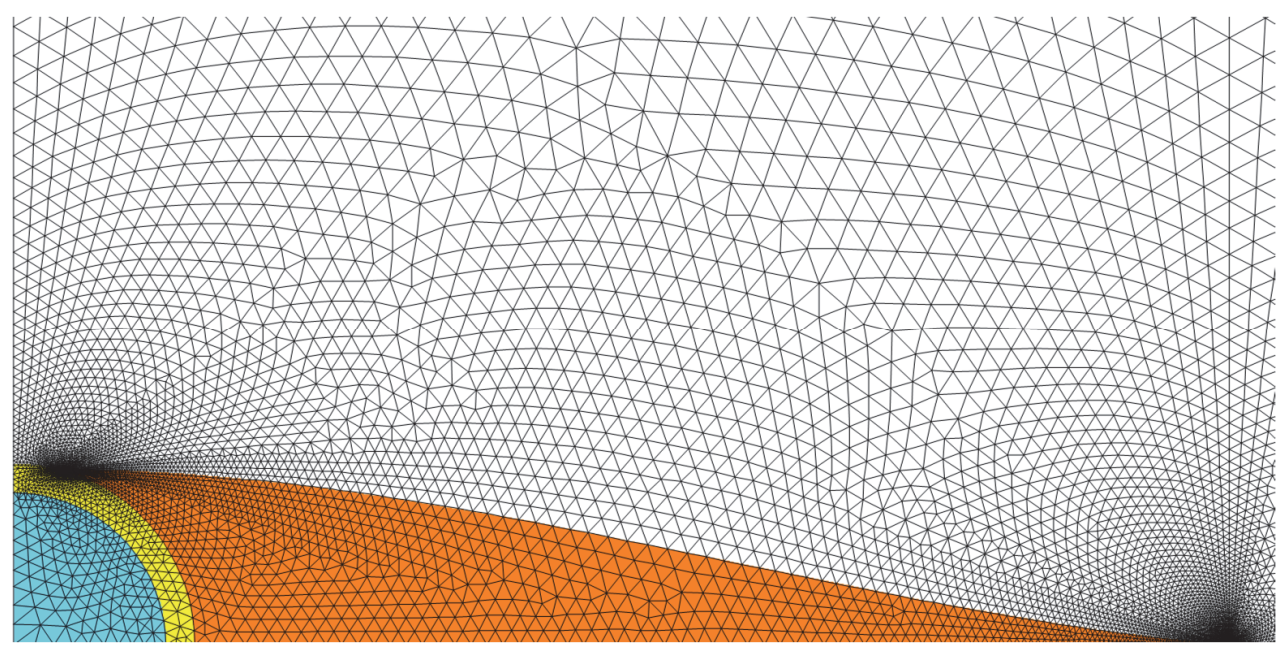

Figure 8: Finite element mesh of the model with embedded optical fiber and resin pocket.

The purpose of numerical experiments is to calculate the strains $\varepsilon_{1}, \varepsilon_{2}, \varepsilon_{3}$ in the optical fiber under different loading options, and to calculate the values $\Delta \lambda_{1} / \lambda^{*}$ and $\Delta \lambda_{2} / \lambda^{*}$ according to Eq. (2). Wherein, the coincidence of the strain $\varepsilon_{3}$ 
and the corresponding strain in the material in the vicinity of the fiber is controlled in the calculations. Further, the values $\Delta \lambda_{1} / \lambda^{*}$ and $\Delta \lambda_{2} / \lambda^{*}$ are taken as experimental data to calculate the strain $\varepsilon_{3}^{\text {exp }}$ along the fiber based on the Eq. (4). Then the error between the strain $\varepsilon_{3}$ and $\varepsilon_{3}^{\text {exp }}$ is estimated. In Tab. 3 the values $\varepsilon_{1}^{*}, \varepsilon_{2}^{*}, \varepsilon_{3}^{*},\left(\Delta \lambda_{1} / \lambda^{*}\right)^{*},\left(\Delta \lambda_{2} / \lambda^{*}\right)^{*}$ and the magnitude of the errors $\delta_{1}$ and $\delta_{2}$ are shown. Here $\varepsilon_{1}^{*}=\varepsilon_{1} / u_{o}, \varepsilon_{2}^{*}=\varepsilon_{2} / u_{o}, \quad \varepsilon_{3}^{*}=\varepsilon_{3} / u_{o}$; $\left(\frac{\Delta \lambda_{1}}{\lambda}\right)^{*}=\left(\frac{\Delta \lambda_{1}}{\lambda}\right) / u_{o},\left(\frac{\Delta \lambda_{2}}{\lambda}\right)^{*}=\left(\frac{\Delta \lambda_{2}}{\lambda}\right) / u_{o}$. The error in the calculation of strain based on the Eq. (4) is determined as follows: $\delta_{1}=\left(\varepsilon_{3}^{\exp _{1}}-\varepsilon_{3}\right) / \varepsilon_{3} \cdot 100 \%, \delta_{2}=\left(\varepsilon_{3}^{\exp _{2}}-\varepsilon_{3}\right) / \varepsilon_{3} \cdot 100 \%$, where $\varepsilon_{3}^{\exp _{1}}=\frac{1}{k} \cdot \frac{\Delta \lambda_{1}}{\lambda^{*}}, \varepsilon_{3}^{\exp _{2}}=\frac{1}{k} \cdot \frac{\Delta \lambda_{2}}{\lambda^{*}}$. Tab. 3 also shows the values of $k_{1}$ and $k_{2}$, which, according to the formulas $\Delta \lambda_{1} / \lambda^{*}=k_{1} \cdot \varepsilon_{3}, \Delta \lambda_{2} / \lambda^{*}=k_{2} \cdot \varepsilon_{3}$, provide reliable strain values.

Layered model with the formation of a resin pocket [90/90]

$\begin{array}{lccccccccc} & \varepsilon_{1}^{*} & \varepsilon_{2}^{*} & \varepsilon_{3}^{*} & \left(\Delta \lambda_{1} / \lambda^{*}\right)^{*} & \left(\Delta \lambda_{2} / \lambda^{*}\right)^{*} & k_{1} & \delta_{1} \% & k_{2} & \delta_{2} \% \\ u_{x}=u_{0} & -74.01 & -94.32 & 500.00 & 389.88 & 393.09 & 0.78 & 0.03 & 0.79 & 0.79 \\ u_{y}=u_{0} & 106.31 & -84.90 & -22.26 & -35.46 & -5.18 & 1.59 & 104.22 & 0.23 & 70.16 \\ u_{z}=u_{0} & 113.62 & 10.08 & -35.27 & -42.65 & -59.05 & 1.21 & 55.05 & 1.67 & 114.66\end{array}$

Homogeneous model with the formation of a resin pocket

$\begin{array}{ccccccccrr} & \varepsilon_{1}^{*} & \varepsilon_{2}^{*} & \varepsilon_{3}^{*} & \left(\Delta \lambda_{1} / \lambda^{*}\right)^{*} & \left(\Delta \lambda_{2} / \lambda^{*}\right)^{*} & k_{1} & \delta_{1} \% & k_{2} & \delta_{2} \% \\ u_{x}=u_{0} & -74.15 & -94.17 & 500.00 & 389.90 & 393.07 & 0.78 & 0.02 & 0.79 & 0.79 \\ u_{y}=u_{0} & 91.54 & -53.13 & -20.02 & -35.96 & -13.04 & 1.80 & 130.28 & 0.65 & 16.46 \\ u_{z}=u_{0} & 108.86 & 10.29 & -30.49 & -43.47 & -59.08 & 1.43 & 82.76 & 1.94 & 148.40\end{array}$

Layered model without the formation of a resin pocket $[0 / 0]$

$\begin{array}{ccccccccrr} & \varepsilon_{1}^{*} & \varepsilon_{2}^{*} & \varepsilon_{3}^{*} & \left(\Delta \lambda_{1} / \lambda^{*}\right)^{*} & \left(\Delta \lambda_{2} / \lambda^{*}\right)^{*} & k_{1} & \delta_{1} \% & k_{2} & \delta_{2} \% \\ u_{x}=u_{0} & -64.57 & -96.30 & 500.00 & 387.43 & 392.45 & 0.77 & 0.66 & 0.78 & 0.63 \\ u_{y}=u_{0} & 86.79 & -27.76 & -14.02 & -31.33 & -13.19 & 2.23 & 186.51 & 0.94 & 20.64 \\ u_{z}=u_{0} & 92.21 & 7.86 & -23.99 & -31.22 & -44.58 & 1.30 & 66.83 & 1.86 & 138.22\end{array}$

Homogeneous model without the formation of a resin pocket

\begin{tabular}{lccccccccc} 
& $\varepsilon_{1}^{*}$ & $\varepsilon_{2}^{*}$ & $\varepsilon_{3}^{*}$ & $\left(\Delta \lambda_{1} / \lambda^{*}\right)^{*}$ & $\left(\Delta \lambda_{2} / \lambda^{*}\right)^{*}$ & $k_{1}$ & $\delta_{1} \%$ & $k_{2}$ & $\delta_{2} \%$ \\
$u_{x}=u_{0}$ & -74.30 & -94.93 & 500.00 & 390.04 & 393.31 & 0.78 & 0.01 & 0.79 & 0.85 \\
$u_{y}=u_{0}$ & 403.99 & -147.25 & -18.58 & -105.69 & -18.39 & 5.69 & 629.14 & 0.99 & 26.85 \\
$u_{z}=u_{0}$ & 104.11 & -13.96 & -28.63 & -25.16 & -43.86 & 0.88 & 12.65 & 1.53 & 96.38 \\
\hline
\end{tabular}

Table 3: The results of the evaluation of the limits of applicability of relations (4) on a model of a cube with integrated optical fiber. 
For clarity, the dependences of $k_{1}$ and $k_{2}$ with respect to the plane $k=0.78$ by varying $\ln \left(\varepsilon_{2} / \varepsilon_{3}\right), \ln \left(\varepsilon_{1} / \varepsilon_{3}\right)$ are presented in Fig. 9.
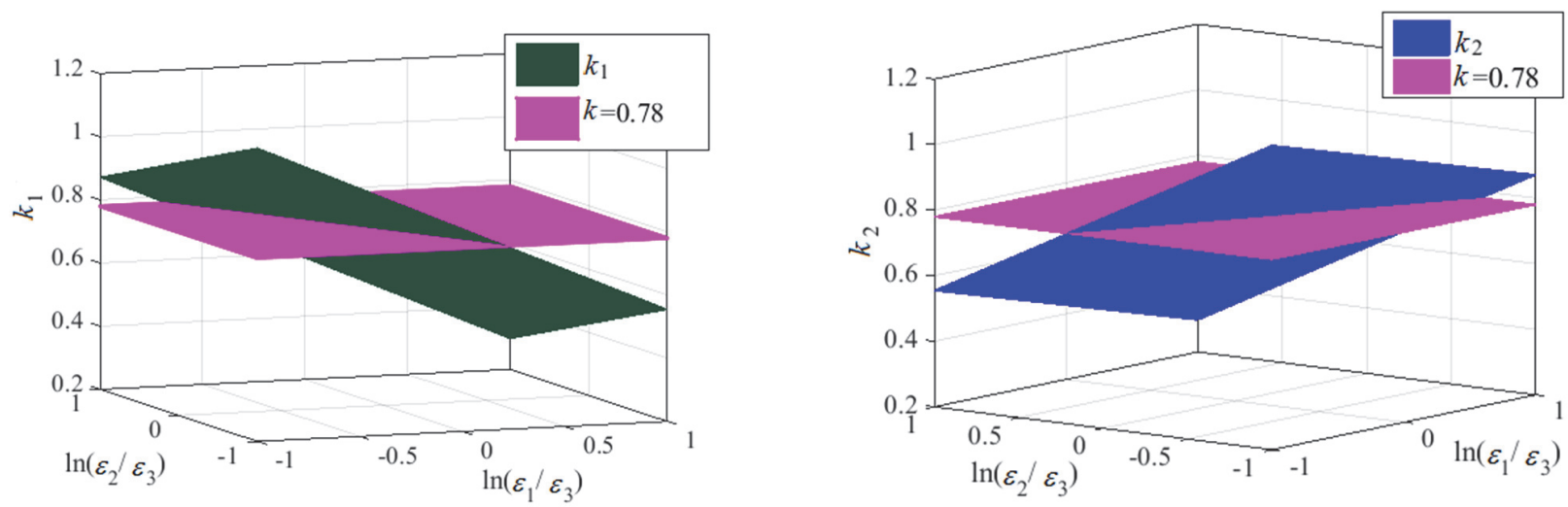

Figure 9: Dependences of $k_{1}$ and $k_{2}$ on the components of the strain tensor.

The obtained results allow us to conclude that the use of Bragg gratings written in a single-mode optical fiber provides acceptable information about the component of the strain tensor along the fiber, only in case when the sensor is located in the material zone, where this component of strain is predominant. The dependences given in Fig. 9 allow us to estimate the error in the calculation of the strain for different ratios of the strain values $\varepsilon_{1}$ and $\varepsilon_{2}$.

\section{SIMULATION OF FOSS EMBEDDED IN PCM WITH A CAPILLARY ON THE BRAGG GRATING}

$\mathrm{O}$

ne of the directions for obtaining reliable information about the strains measured by FBG sensors embedded into PCM is associated with a change in the conditions of interaction with the material of the optical fiber section with the Bragg grating. In a constructive implementation, this can be achieved by using an additional coating around the fiber in the Bragg grating zone (Fig. 10).

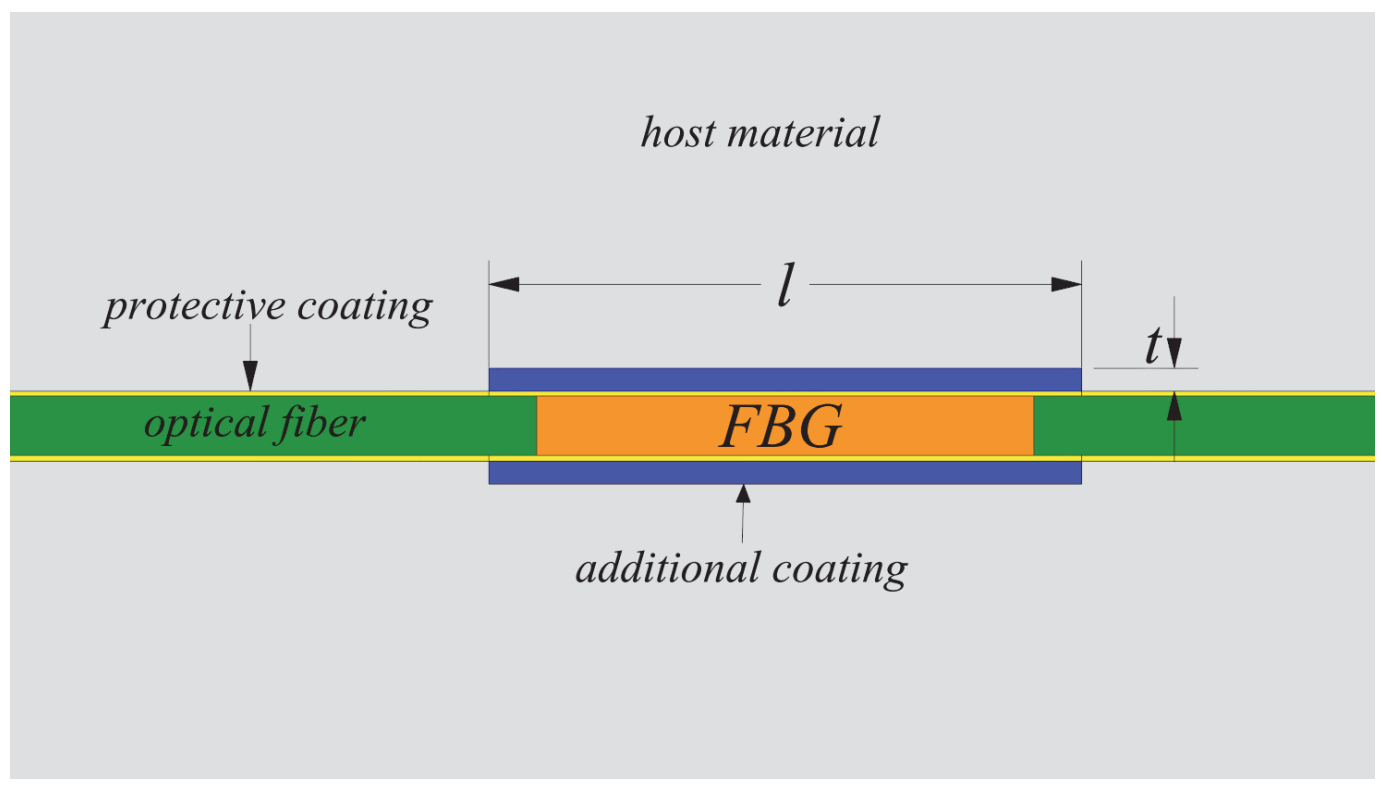

Figure 10: Cross-section of the sample with FOSS based on the Bragg grating and capillary. 
The geometrical dimensions of such a coating are determined by the thickness $t$ and the length $l$. Within the framework of the problem statement under consideration, an additional coating must ensure the fulfillment of two conditions: the realization of a uniaxial stress state in the Bragg grating section and the coincidence of the longitudinal strain component in the fiber and in the material zone adjacent to the fiber.

Numerical simulation allows us to estimate the possibility of achieving the goal and determine the parameters of an additional coating for the corresponding PCM and optical fiber. A problem of stress-strain state evaluation on a model of a cube with embedded optical fiber with additional coating is considered (Fig. 11). A variant of the calculation scheme with resin pocket is not presented, since its consideration does not affect the magnitude of the strain tensor component along the optical fiber.

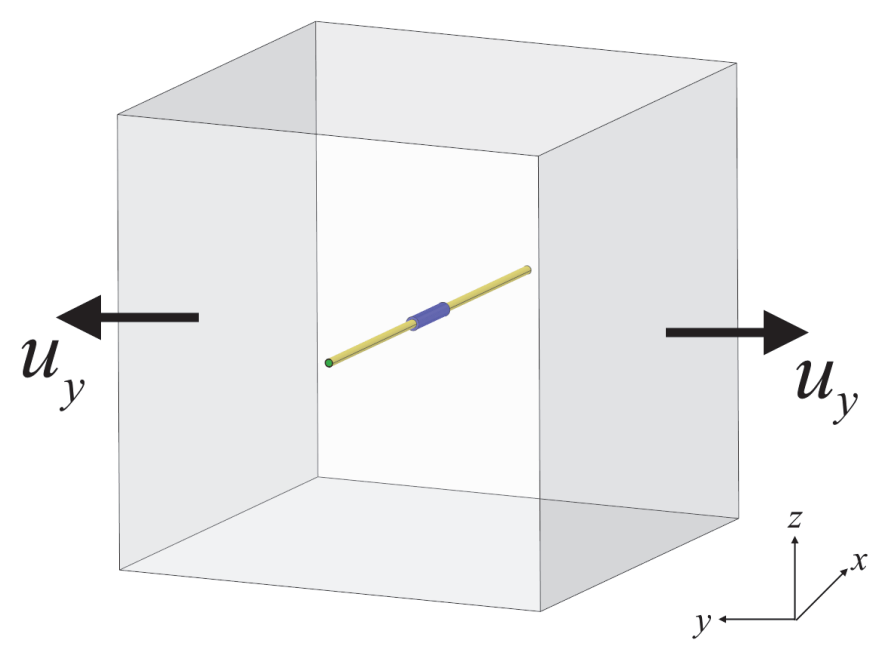

Figure 11: Cube with embedded optical fiber and additional coating.

Dimensions of the cube significantly exceed the length of the additional coating. Based on the results of the previous chapter, the most unfavorable loading option is considered, in which external forces operate in the plane of maximum stiffness of the PCM and the direction perpendicular to the fiber.

In order to estimate the parameters of the coating that ensure the fulfillment of the condition of a uniaxial stress state in the zone of the Bragg grating of the optical fiber, calculations were performed for various combinations of Poisson's ratio and the ratio of the elastic moduli of the optical fiber and the additional coating. Calculations have shown that the goal can be achieved only if there is a cavity between the PCM and the optical fiber. Technologically, this means using a capillary in the Bragg grating zone [15].

With the considered mechanical characteristics of PCM, it is not possible to ensure the coincidence of the corresponding strain in the material and the fiber. So, with a capillary thickness and its length equal to 0.3 and 10 fiber diameters, respectively, the difference between the corresponding strains in the PCM and the optical fiber in the middle cross section along the length of the capillary is $4-5$ times.

The change in the mechanical characteristics of the PCM showed that it is possible to achieve the conditions of coincidence of the strains when using a capillary with a circular cross section with the isotropic properties of the material. For anisotropic materials, these conditions can be achieved by using a capillary with an elliptical cross section (Fig. 12).

The results of calculations of the strains $\varepsilon_{x} / u_{y}, \varepsilon_{y} / u_{y}, \varepsilon_{z} / u_{y}$ in an optical fiber, in the middle across the length cross section of the capillary for various relations between the ellipse's semi axes $a(a=0.16 \mathrm{~mm}), b$ and ratios of the capillary length $l$ to the optical fiber diameter $d$ are presented in Tab. 4 for materials whose properties are given in Tabs. 1 and 2. The analysis of these results should be carried out taking into account the strain value $\varepsilon_{x}$ in PCM equal to $4.37 \cdot 10^{-3}$. For the data in the table, it should be noted that the condition $\varepsilon_{y}=\varepsilon_{z}=-v \varepsilon_{x}$, where $\nu$ is the Poisson's ratio of the optical fiber material is fulfilled up to $0.22 \%$. The results show that if $a / b=0.6$, it is possible to achieve an acceptable coincidence of the strain along the fiber in the PCM and the optical fiber.

The obtained values of $l$ show that when using such a capillary, its length should be 20-40 diameters larger than the Bragg grating length to ensure that the strain in the material and the fiber coincides along the entire Bragg grating length. The 
performed calculations show the possibility of determining by the methods of numerical simulation for specific characteristics of PCM and optical fiber of the capillary sizes that ensure the fulfillment of the set conditions. Technological aspects of the implementation of the results obtained in this paper are not considered.

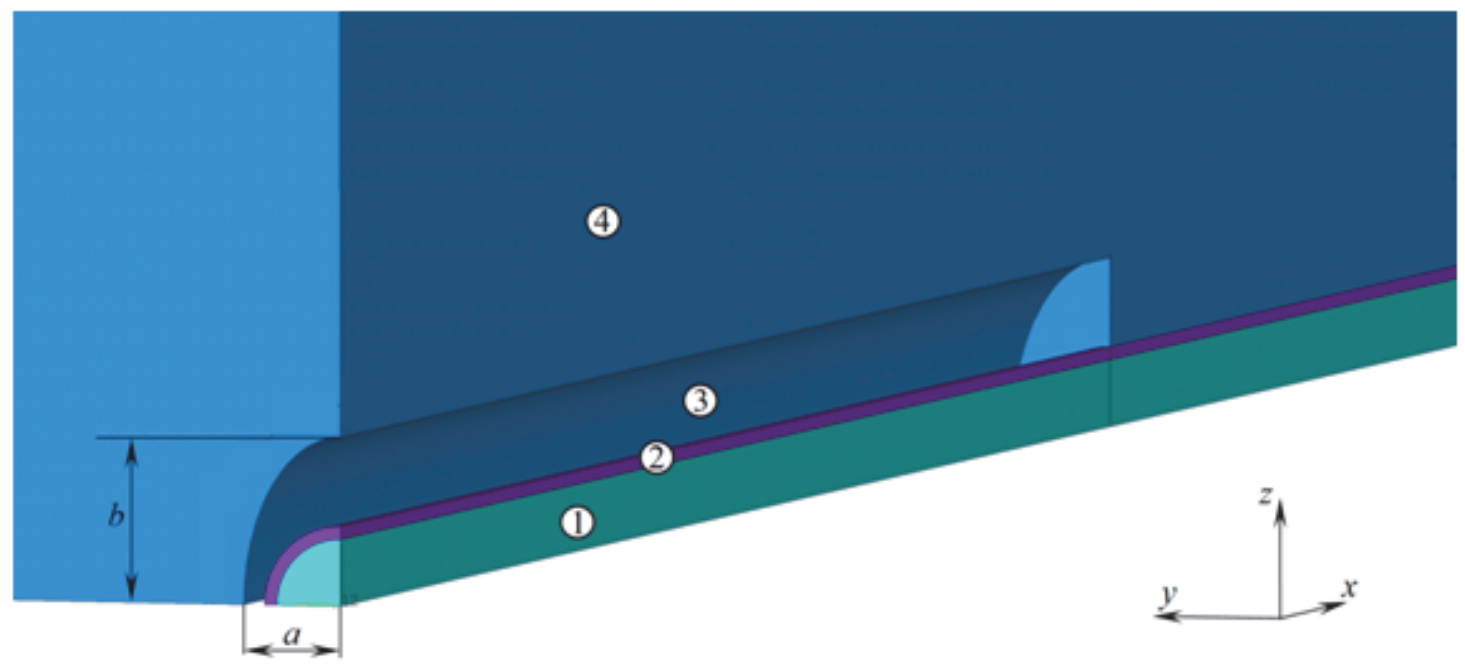

Figure 12: The scheme of a polymer composite material with an embedded optical fiber and a cavity with an elliptical cross section: optical fiber (1); protective polyimide coating of optical fiber (2); cavity (3); composite material (4).

\begin{tabular}{ccccc}
\hline$\varepsilon_{x} / u_{y}$ & $\varepsilon_{y} / u_{y}$ & $\varepsilon_{z} / u_{y}$ & $a / b$ & $k=l / d$ \\
-0.002653 & 0.000452 & 0.000452 & 1 & 10 \\
-0.001943 & 0.000330 & 0.000330 & 2 & 10 \\
-0.005044 & 0.000858 & 0.000858 & 0.5 & 10 \\
-0.004837 & 0.000822 & 0.000822 & 0.5 & 20 \\
-0.00399 & 0.000678 & 0.000678 & 0.75 & 10 \\
-0.00419 & 0.000712 & 0.000712 & 0.6 & 10 \\
-0.004348 & 0.000739 & 0.000739 & 0.6 & 20 \\
-0.004372 & 0.000743 & 0.000743 & 0.6 & 40 \\
\hline
\end{tabular}

Table 4: The results of the calculation of strains in the optical fiber with different capillary geometries.

\section{CONCLUSIONS}

he analysis of the possibility of obtaining reliable strain values in the PCM based on the use of FBG sensor written in a single-mode optical fiber and embedded in the material is carried out. It is noted that for this type of sensors direct relation between the longitudinal strain of the FBG, which with good contact of the sensor with the PCM coincides with the corresponding strain in the material, and the physical quantity recorded by the sensor takes place only with a uniaxial stress state in the fiber. A numerical experimental technique is presented. Using the assumption about the nature of stresses in a fiber, it is possible to estimate the error in calculating the strain values of an optical fiber in the FBG zone based on the change in the wavelength of the reflected spectrum measured by the sensor.

For the model object of PCM with embedded FOSS, an analysis of the strain fields in the fiber and material under different loading options is performed. Calculations based on the finite element method were carried out for two PCM models: a layered model and a homogeneous medium model. The model takes into account the possibility of a resin pocket in the vicinity of the fiber. Different options for the optical fiber location between the layers [0/0], when the resin pocket is absent, and between the layers [90/90], when the resin pocket is formed, were analyzed. 
Obtained results make it possible to estimate the error of the values of the strain tensor component in the fiber along its length based on the values of the physical quantity measured by the sensor, which is the result of numerical calculations within the framework of the considered technique. The combination of the achieved numerical results makes it possible to conclude that for obtaining, with an acceptable error, the values of the measured strains, it is necessary that the sensors should be located in the material zone, where the strain component along the fiber is predominant.

A numerical simulation was performed to determine the parameters of the coating around the FBG, which provides a uniaxial stress state in the FBG and the coincidence of the strain values along the fiber directly in the fiber and in the material zone adjacent to the fiber. It is established that the uniaxial stress state is reached only in the presence of a capillary (cavity) between the PCM and the optical fiber. The condition of coincidence of the corresponding strains in the material and the fiber at the circular cross-section of the capillary is ensured only with the isotropic properties of PCM. It is shown that for anisotropic PCM the fulfillment of the condition of coincidence of strains can be achieved with an elliptical capillary cross section. The specific dimensions of the capillary are found by numerical analysis for the corresponding characteristics of the PCM and the optical fiber.

\section{ACKNOWLEDGEMENTS}

his study was supported by Russian Science Foundation (project No.15-19-00243).

\section{REFERENCES}

[1] Gebremichael, Y.M., Li, W., Boyle, W.J.O., Meggitt, B.T., Grattan, K.T. V., McKinley, B., Fernando, G.F., Kister, G., Winter, D., Canning, L., Luke, S. (2005). Integration and assessment of fibre Bragg grating sensors in an all-fibre reinforced polymer composite road bridge, Sensors Actuators, A Phys., 118(1), pp. 78-85,

DOI: 10.1016/j.sna.2004.08.005.

[2] Lee, J.-R., Ryu, C.-Y., Koo, B.-Y., Kang, S.-G., Hong, C.-S., Kim, C.-G. (2003). In-flight health monitoring of a subscale wing using a fiber Bragg grating sensor system, Smart Mater. Struct., 12, pp. 147-155,

DOI: 10.1088/0964-1726/12/1/317.

[3] Ghoshal, A., Ayers, J., Gurvich, M., Urban, M., Bordick, N. (2015). Experimental investigations in embedded sensing of composite components in aerospace vehicles, Compos. Part B Eng., 71, pp. 52-62,

DOI: 10.1016/j.compositesb.2014.10.050.

[4] Wymore, M.L., Van Dam, J.E., Ceylan, H., Qiao, D. (2015). A survey of health monitoring systems for wind turbines, Renew. Sustain. Energy Rev., 52(1069283), pp. 976-990, DOI: 10.1016/j.rser.2015.07.110.

[5] Sierra-Pérez, J., Torres-Arredondo, M.A., Güemes, A. (2016). Damage and nonlinearities detection in wind turbine blades based on strain field pattern recognition. FBGs, OBR and strain gauges comparison, Compos. Struct., 135, pp. 156-166, DOI: 10.1016/j.compstruct.2015.08.137.

[6] Hong, C.Y., Zhang, Y.F., Zhang, M.X., Leung, L.M.G., Liu, L.Q. (2016). Application of FBG sensors for geotechnical health monitoring, a review of sensor design, implementation methods and packaging techniques, Sensors Actuators, A Phys., , pp. 184-197, DOI: 10.1016/j.sna.2016.04.033.

[7] Kersey, A.D., Davis, M.A., Patrick, H.J., LeBlanc, M., Koo, K.P., Askins, C.G., Putnam, M.A., Friebele, E.J. (1997). Fiber grating sensors, J. Light. Technol., 15(8), pp. 1442-1463, DOI: 10.1109/50.618377.

[8] Luyckx, G., Voet, E., Lammens, N., Degrieck, J. (2010). Strain Measurements of Composite Laminates with Embedded Fibre Bragg Gratings: Criticism and Opportunities for Research, Sensors, 11(1), pp. 384-408, DOI: $10.3390 / \mathrm{s} 110100384$.

[9] Di Sante, R. (2015). Fibre Optic Sensors for Structural Health Monitoring of Aircraft Composite Structures: Recent Advances and Applications, Sensors, 15(8), pp. 18666-18713, DOI: 10.3390/s150818666.

[10] Majumder, M., Gangopadhyay, T.K., Chakraborty, A.K., Dasgupta, K., Bhattacharya, D.K. (2008). Fibre Bragg gratings in structural health monitoring - Present status and applications, Sensors and Actuators A-Physical, 147(1), pp. 150-164, DOI: 10.1016/j.sna.2008.04.008.

[11] Fan, Y., Kahrizi, M. (2005). Characterization of a FBG strain gage array embedded in composite structure, Sensors Actuators, A Phys., 121(2), pp. 297-305, DOI: 10.1016/j.sna.2005.01.021. 
[12] Sonnenfeld, C., Luyckx, G., Sulejmani, S., Geernaert, T., Eve, S., Gomina, M., Chah, K., Mergo, P., Urbanczyk, W., Thienpont, H., Degrieck, J., Berghmans, F. (2015). Microstructured optical fiber Bragg grating as an internal threedimensional strain sensor for composite laminates, Smart Mater. Struct., 24(5), pp. 055003, DOI: 10.1088/0964-1726/24/5/055003.

[13] Sonnenfeld, C., Sulejmani, S., Geernaert, T., Eve, S., Lammens, N., Luyckx, G., Voet, E., Degrieck, J., Urbanczyk, W., Mergo, P., Becker, M., Bartelt, H., Berghmans, F., Thienpont, H. (2011). Microstructured optical fiber sensors embedded in a laminate composite for smart material applications, Sensors, 11(3), pp. 2566-2579, DOI: $10.3390 / \mathrm{s} 110302566$.

[14] Luyckx, G., Voet, E., DeWaele, W., Degrieck, J. (2010). Multi-axial strain transfer from laminated CFRP composites to embedded Bragg sensor: I. Parametric study, Smart Mater. Struct., 19(10), DOI: 10.1088/0964-1726/19/10/105017.

[15] Voet, E., Luyckx, G., De Waele, W., Degrieck, J. (2010). Multi-axial strain transfer from laminated CFRP composites to embedded Bragg sensor: II. Experimental validation, Smart Mater. Struct., 19(10), pp. 105018, DOI: 10.1088/0964-1726/19/10/105018.

[16] Li, Y., Wen, C., Sun, Y., Feng, Y., Zhang, H. (2014). Capillary encapsulating of fiber Bragg grating and the associated sensing model, Opt. Commun., 333, pp. 92-8, DOI: 10.1016/j.optcom.2014.07.068.

[17] Lammens, N., Luyckx, G., Degrieck, J., De Waele, W. (2011). Experimental determination of the multi-axial strain transfer from CFRP-laminates to embedded Bragg sensor, Smart Struct. Mater. 5th ECCOMAS Themat. Conf. Proc., pp. 482-485.

[18] Wagreich, R.B., Atia, W.A., Singh, H., Sirkis, J.S. (1996). Effects of diametric load on fibre Bragg gratings fabricated in low birefringent fibre, Electron. Lett., 32(13), pp. 1223, DOI: 10.1049/el:19960806.

[19] Matveenko, V.P., Kosheleva, N.A., Shardakov, I.N., Voronkov, A.A. (2018). Temperature and strain registration by fibre-optic strain sensor in the polymer composite materials manufacturing, Int. J. Smart Nano Mater., 9(2), pp. 99110, DOI: 10.1080/19475411.2018.1450791.

[20] Lammens, N., Luyckx, G., Voet, E., Van Paepegem, W., Degrieck, J. (2015). Finite element prediction of resin pocket geometry around embedded optical fiber sensors in prepreg composites, Compos. Struct., 132, pp. 825-832, DOI: 10.1016/j.compstruct.2015.07.003.

[21] Shivakumar, K., Bhargava, A. (2005). Failure mechanics of a composite laminate embedded with a fiber optic sensor, J. Compos. Mater., 39(9), pp. 777-798, DOI: 10.1177/0021998305048156. 